\title{
Towards a global list of accepted species I. Why taxonomists sometimes disagree, and why this matters
}

\author{
Kevin R. Thiele ${ }^{1,2}$ (1) $\cdot$ Stijn Conix ${ }^{3}$ (1) $\cdot$ Richard L. Pyle $^{4}$ (D) $\cdot$ Saroj K. Barik $^{5}$ (D) $\cdot$ Les Christidis $^{6}$ (D) Mark John Costello ${ }^{7,8}$. \\ Peter Paul van Dijk ${ }^{9}$ - Paul Kirk ${ }^{10}$. Aaron Lien ${ }^{11}$. Scott A. Thomson ${ }^{12}$ (D) Frank E. Zachos $^{13,14,15}$. \\ Zhi-Qiang Zhang ${ }^{16,17}$ (D) Stephen T. Garnett ${ }^{18}$ (D)
}

Received: 30 January 2021 / Accepted: 18 May 2021 / Published online: 13 July 2021

(C) Gesellschaft für Biologische Systematik 2021

\begin{abstract}
Taxonomy - the delimitation, naming, classification and documentation of species and other taxa-is an often-misunderstood discipline. Complex and at times contested, taxonomy occupies a sometimes discomforting intermediate position on a continuum from descriptive to hypothetico-deductive science. Two aspects of taxonomy that are striking to many observers and users are the degree to which taxonomists often disagree, and the degree of taxonomic revisionism (the replacement of one taxonomic classification with another, exemplified by the phrase 'taxonomists are always changing the names of things'). Disagreements between taxonomists do not usually indicate taxonomic confusion or chaos, but rather often represent valid disagreements over the best, most effective and most meaningful way to interpret, describe and classify one of the most complex systems that scientists seek to describe and characterise - the patterns of variation of life on Earth. One way to partially manage disagreements among taxonomists is to develop a mechanism to synthesise the flux of taxonomic activity into agreed, broadly accepted, authoritative and scientifically robust global lists of the world's species and other taxa. A sound understanding of some aspects of the nature of taxonomy is needed to appreciate the opportunities, complexities and limitations of the development and maintenance of such lists.
\end{abstract}

This article is part of the Special Issue and Topical Collection Towards a global list of accepted species

Kevin R. Thiele

kevin.thiele@science.org.au

1 Australian Academy of Science, Ian Potter House, 9 Gordon Street, Acton ACT 2601, Australia

2 The University of Western Australia, 35 Stirling Highway, Perth, WA 6009, Australia

3 Centre for Logic and Philosophy of Science, KU Leuven, Leuven, Belgium

4 Bernice Pauahi Bishop Museum, Honolulu, HI, USA

5 CSIR-National Botanical Research Institute, Rana Pratap Marg, Lucknow, India

6 Southern Cross University, Coffs Harbour, Australia

7 School of Environment, University of Auckland, Auckland, New Zealand

8 Faculty of Biosciences and Aquaculture, Nord University, Bodø, Norway
9 Global Wildlife Conservation, Austin, TX, USA

10 Royal Botanic Gardens Kew, Richmond, UK

11 School of Natural Resources and the Environment, University of Arizona, Tucson, AZ, USA

12 Chelonian Research Institute, Oviedo, FL, USA

13 Natural History Museum Vienna, Vienna, Austria

14 Department of Evolutionary Biology, University of Vienna, Vienna, Austria

15 Department of Genetics, University of the Free State, Bloemfontein, South Africa

16 Manaaki Whenua-Landcare Research, Auckland, New Zealand

17 School of Biological Sciences, The University of Auckland, Auckland, New Zealand

18 Research Institute for the Environment and Livelihoods, Charles Darwin University, Darwin, Australia 
Keywords Taxonomy $\cdot$ Map-making $\cdot$ Taxonomic disagreement $\cdot$ Global lists

\section{Introduction}

Taxonomy - the delimitation, naming, classification and documentation of species and other taxa - is a foundational component of biology. It underpins, enables and provides essential tools for understanding the nature of life on Earth. In doing so, taxonomists deal with one of the most complex systems known-living organisms and their evolutionary and ecological interactions and histories.

Taxonomy is also critically important to our understanding of, and ability to study, manage and conserve, biodiversity. Progress in a wide range of disciplines including ecology, genetics, physiology, biochemistry and the earth sciences rely on the structured framework of life that taxonomy provides. A wide range of other important societal activities including conservation, agriculture, biosecurity, medicine, trade and bioprospecting also depend substantially on a robust, dependable and scientifically valid taxonomy, as do many human connections to the natural world.

Taxonomies ${ }^{1}$ in the broad sense-systems of naming and classifying items of interest - are common throughout science and other domains of knowledge. Many taxonomies in science are straightforward and relatively free of controversy and complexity. Such taxonomies occupy the ends of a continuum from high objectivity to high subjectivity. The taxonomy of chemical elements, for example, is almost entirely objective - once chemists agreed that elements should be classified by atomic number, classifying and identifying them became a matter of largely incontrovertible fact. At the other end of the continuum, the taxonomy of colours is highly subjective, based as it is on a clearly arbitrary division of the continuous spectrum of light into named units. Few people are likely to contest the taxonomy of chemistry, or to bother contesting the taxonomy of colours. These non-controversial taxonomies are also characterised by the relative simplicity of the domains they seek to describe.

The taxonomy of biodiversity, by contrast, occupies a middle ground on this continuum, somewhere between incontrovertible and arbitrary (different practitioners and observers of taxonomy are likely to differ as to where exactly on this continuum they would place it, but its 'precise' position is immaterial to this paper). In this middle ground, there is enough subjectivity to make taxonomy contestable, and enough objectivity to make contestation worthwhile. For this reason, the taxonomy of species can sometimes be a highly contested

\footnotetext{
${ }^{1}$ In this paper, the word 'taxonomy' (singular and when not qualified) is used in a specific sense as the taxonomy of biodiversity. In a broader sense, many disciplines require taxonomies, including medicine and anatomy, chemistry, astronomy, physics, computer science and many domains outside of science. When the word 'taxonomies' (plural) is used, it refers to this broader sense.
}

space, an unsurprising outcome given the complexity of biodiversity and its patterns of variation. Nevertheless, modern systems of taxonomy, nomenclature and classification have remained fundamentally unaltered for a quarter of a millennium, underscoring the importance and enduring need for a stable and robust biological taxonomy.

This paper is one in a series that describe various aspects of a global initiative to compile, curate and maintain a widely accepted consensus list and classification of all the world's species and other taxa, an avowedly practical (though decidedly non-trivial) endeavour. The papers attempt to grapple with some of the complexities and nuances of the task. This first paper aims to set the scene by describing some of the aspects of taxonomy that make such an enterprise possible and useful, and at the same time difficult and necessarily imperfect. We first discuss the nature of taxonomy, then the reasons why taxonomists sometimes disagree and the reasons why these disagreements are both unavoidable and understandable.

\section{What type of science is taxonomy?}

Natural science - the description, prediction and understanding of natural phenomena - encompasses a wide range of disciplines. An imperfect division of science often differentiates its activities into 'descriptive' or 'observational', and 'hypothesis-driven' or 'hypothetico-deductive' (Casadevall \& Fang, 2008). The former two terms are often used in a pejorative sense (made more so by the common prefix 'merely') and regarded as less important, less valuable and sometimes even less 'scientific', than the latter two. Of course, much of science is a complex (and often iterative) mix of mutually supportive description and hypothesis, and there are dangers in reducing the complement of either.

In addition to having a high component of descriptiveness, several other characteristics of taxonomy cause some to overlook its importance: it is foundational, it has a long history and it is sometimes (but by no means always) relatively low-tech. Foundations are almost always less glamorous than the superstructures built upon them, a long history is often misconstrued as 'old-fashioned' and high-tech is often seen as a good in itself and, in the popular imagination at least, as somehow 'more scientific' than low-tech.

Responding to some of these impediments, taxonomists are sometimes at pains to paint the discipline as hypothetico-deductive, with taxa as hypotheses (e.g. Pante et al., 2015; Raposo et al., 2017; Sluys, 2013). However, a problem with this interpretation lies with the baggage that accompanies these terms. In particular, hypotheses are often judged using 
stringent concepts such as falsifiability: hypotheses are tested, and those that fail the test are deemed false. Applying the concept of falsifiability to taxonomy implies that taxa (species etc.) must be either true or false (and cannot be both, or neither).

Hypothesis-testing certainly can play a role in taxonomy, and in some cases a taxon-as-hypothesis can be determined to be objectively false, especially when it is resolved using a strictly defined method and the method can be demonstrated to be either false or incorrectly interpreted. However, only a very small minority of taxonomic treatments incorporate such strictly defined methods and analyses. Stringent concepts fail to account for an important nuance that permeates the vast majority of taxonomy: two or more different taxonomic delimitations or classifications for the same set of primary data (organisms and their patterns of variation) may both be 'true' (or at least, neither may be objectively 'false'). Consider a case of two related but distinct metapopulations that differ in some traits or combination of traits (morphological, genetic etc.). One taxonomist may decide that the differences are significant enough to recognise two species while another may decide that the differences are not sufficient and will recognise a single species. A stringent claim that only one of these two alternate 'hypotheses' can be true fails to do justice to the very real latitude of such decision-making.

\section{Taxonomy as 'map-making'}

Better analogies are available for taxonomy, free from the tensions caused when it is forced into a hypotheticodeductive straitjacket with an over-emphasis on apparent objectivity. One is taxonomy as an information science (see Kendig \& Witteveen, 2020, and references cited therein). Another is taxonomy as map-making (see also Kitcher, 1984, 2001; O'Hara, 1993). Just as cartographers examine patterns in landscapes and from these draw and label simplified maps to represent them, so taxonomists examine patterns in nature and from these delimit and label (name) taxa, thus contributing to a 'map' that others use to navigate biodiversity. A map is a conceptual model of a geographic area, and a taxon is a conceptual model of an enduring evolutionary lineage. The patterns of variation that taxonomists study are bewilderingly complex, and it is the task of taxonomists to abstract this complexity into a more or less simple system that others can follow and use without becoming themselves bewildered.

Thinking about taxonomy as map-making helps avoid two common pitfalls that cause confusion for non-taxonomists (and even some taxonomists) in thinking about taxonomy. The first pitfall derives from thinking about taxonomy as pure hypothesis-generation, as discussed above. Taxonomy-asmap-making helps resolve the apparent paradox that two taxonomists can derive two different taxonomies for the same portion of biodiversity, and neither can be falsified, because there may be multiple valid ways to abstract a complex pattern into a 'map' and to name its component parts.

The second pitfall is thinking of taxa as 'real' items in nature that are 'discovered' by taxonomists, implying that the taxa are 'out there in nature' before they are 'found'. A more accurate and only slightly more cumbersome rephrasing would be to say that a taxon is 'delimited', 'resolved' or 'recognised' as new (Zachos et al., 2020), a phraseology that gives more agency to the taxonomist than the more passive action of 'finding'. This pitfall is an example of the common epistemological problem expressed in the aphorism 'the map is not the territory' (Korzybski, 1933): it mixes the abstraction (taxa) with reality (the patterns of variation in nature), a classic map-territory confusion. Keeping in mind that taxonomists 'resolve' or 'interpret' taxa rather than 'discover' them helps blunt some of the tension caused by disagreements, allowing two taxonomists to disagree with respect to their recognition of taxa while at the same time agreeing about the patterns of variation upon which they are based.

Thinking about taxonomy as map-making also firmly places it in a domain where utility is co-equal with veracity. Two 'maps' may be equally 'true', but one may be more useful, to its users, than the other (Conix, 2018; Dupré, 1999, 2001). Similarly, the power of taxonomic names and classifications resides not just in their attempt to faithfully represent natural biological patterns, but also in the provision of a utilitarian scaffolding which others may use to understand and explore biological complexity. Like maps, capturing too much fine detail can obscure as much as it reveals.

There is nothing simple about either cartography or taxonomy, and the metaphor is apt in that the most useful maps are not necessarily the ones that endeavour to reflect the most precise 'truth' of what they are intended to represent, so much as serve as a means of navigating a complex landscape and function as a practical, generalised and commonly understood contextual framework.

\section{Why taxonomists sometimes disagree}

Given the size and complexity of the task of taxonomically documenting the biodiversity of Earth, it should come as no surprise that taxonomists disagree at times. Disagreement could be seen as some kind of failure, or as a healthy consequence of a vigorous and complex science. We prefer the latter.

Despite the focus on disagreement in this paper, a survey across the whole of biodiversity will show that taxonomists usually agree, even in the most apparently contentious groups such as iconic vertebrates (e.g. McClure et al., 2020). As with 
news, however, disagreements gain more attention than agreement, and appear more frequent than is actually the case.

There are five main types of disagreements among taxonomists: disagreement over the nature of taxonomy itself; over which of many 'species concepts' to adopt; over the boundaries between species and other taxa; over rank; and over the optimum pace of adoption of taxonomic change.

\section{Valid disagreements over the nature of taxonomy}

Disagreements among taxonomists sometimes occur due to the long history of taxonomy. Others have written extensively about the historical development of taxonomy (e.g. Mishler, 2009; Wilkins, 2018; Zachos, 2016); in brief, there have been three distinctly different phases of taxonomy (characterisable as the Linnaean, Jussieuian and Hennigian) separated by two paradigm shifts. From the Linnaean phase of taxonomy we have inherited binomial nomenclature (with its unfortunate consequence that some classification changes also cause name changes), and a ranked classification system. From the Jussieuian, we have inherited a somewhat loose and undefined idea of taxa as 'natural' units, while from the Hennigian we have treethinking, a powerful set of concepts and methods for exploring and representing (in phylogenies) evolutionary descent-with-modification, a key driver of taxonomic patterns.

The paradigm shifts were the realisation by the Jussieuians that artificial classifications (as proposed by Linnaeus) are inadequate for capturing the complexity of biodiversity, and the realisation by the Hennigians that classifying on the basis of overall similarity and difference (as proposed by the Jussieuians) fails to adequately reflect the patterns of descent-with-modification.

As with many other sciences, paradigm shifts are often partial, with leakage of concepts or ways of thinking from previous phases to later ones. While the modern 'taxonomic project' is heavily influenced by Hennig and phylogenetics, some taxonomists retain pre-Hennigian concepts of taxa as clusters of similar organisms rather than branches on a tree. At times, disagreements between taxonomists are due to disagreement over the relative weight given to Hennigian and Jussieuian concepts and practices.

Modern taxonomy is thus somewhat of an amalgam of concepts and practices derived from throughout its long history. For example, a key reason for many taxonomic name changes in recent decades is that modern taxonomy is gradually adjusting classifications made during the Jussieuian phase to reflect more modern interpretations of evolution, and its retention of Linnaean naming conventions means that some of these corrections necessitate name changes. This is unfortunate, but in practice it is unavoidable.

\section{Valid disagreements over 'species concepts'}

Much has been written about the apparent failure by taxonomists, over many decades, to converge on a single, agreed species concept (Ereshefsky, 1992; Wilkins, 2018; Zachos, 2016). Indeed, species concepts have proliferated rather than coalesced in recent decades, with the current (Hennigian) taxonomic project adding several new species concepts to those that had developed under the previous (Jussieuian) project.

This seems like a fundamental problem, but the fact that taxonomists have continued to effectively and efficiently resolve, describe and name new species even without an agreed species concept indicates that, at least operationally, it is not much of a problem at all (except, of course, to philosophers of science).

The analogy of taxonomy-as-map-making helps resolve this seeming paradox. Consider two cartographers independently tasked with drawing a map of a complex mountainous landscape from a set of primary data (gridded height measurements). Both are likely to agree on the pattern of topography (they will draw equivalent topographic lines). But as soon as they start marking peaks on their maps, they may diverge: one may mark a single peak where the other marks two peaks separated by a small saddle. The topographic map is a firstorder abstraction from the primary data, but the delimitation of peaks is a second-order abstraction. Neither map is false, they are just differently marked.

In the same way, the delimitation of taxa from primary data (the patterns of variation among organisms) is a high-order abstraction. And just as the cartographers can still draw highly useful maps even without a rigorous, objective, shared definition of 'peak', so too can taxonomists usefully 'map' biodiversity using concepts (such as species) that defy rigorous, objective definition. The failure to agree on a single, objectively determined species definition is not a failure of taxonomy; rather, it reflects the inadequacy of the question 'what exactly is a species?' (see de Queiroz, 2007, Zachos, 2016 for reviews). The irresoluble problem of taxonomy in this regard is in the attempt to overlay a discrete system (species versus not-species) on a continuous process (evolution) and a pattern of variation that, while 'gappy', is more-or-less continuous and fractal. There will always be friction caused by the exigencies of abstraction, no matter what species concept one chooses.

\section{Valid disagreements over species boundaries}

Disagreements over species boundaries are sometimes caused by disagreements over species concepts, but they often represent valid disagreements between taxonomists who share the same species concept. One taxonomist may decide to draw the boundary (the circumscription) of a species widely and in a way that encompasses a fairly high degree of variation, while 
another may prefer narrower circumscriptions and delimit several species, each with a smaller amount of variation. Given the complexity of the underlying patterns of variation, neither can be said to be right or wrong; they simply have made different, and possibly equally valid, decisions.

A common source of such disagreement, sometimes confused with the application of differing species concepts, is the delimitation of taxa based on different types of data. If one taxonomist delimits species based primarily on patterns of variation in morphology and another delimits species in the same group based primarily on patterns of variation in gene sequences, they may make different decisions and arrive at differing species circumscriptions. Again, neither is necessarily wrong; they are simply working with different aspects of complex patterns of variation. Fortunately, evolutionary processes often generate substantial congruence between patterns of variation within and between genotypes and phenotypes, ameliorating the problem, and taxonomists increasingly attempt to integrate (or at least consider congruence between) independent datasets (e.g. Dayrat, 2005; Padial et al., 2009; Padial et al., 2010; Schlick-Steiner et al., 2010; but see Yeates et al., 2011).

An important but rarely discussed limitation in the traditions of taxonomy exacerbates the tensions caused by the analysis of different patterns of variation: ever since Linnaeus, taxonomists have all been working on the same 'map'. Cartographers avoid this problem - one may draw a map based on topography, another based on underlying geology, another based on built, anthropogenic features such as highways and towns, with no assumption that all should work on a single, synthesis map. This multiplicity of types of cartographic maps, drawn for different purposes and reflecting different underlying realities, contrasts with the unitary nature of taxonomy. It is at least theoretically possible to envisage an explicitly and radically pluralist taxonomy, the product of which is a set of more or less independent, complementary 'maps' of biodiversity, one based on (for example) patterns of variation in morphology, another based on patterns of genetic variation, and yet another based on patterns in ecological traits. That this is rarely even considered (the usual pluralist position is to allow different species concepts in different taxonomic groups, e.g. Dupré, 2001) is an indication of the power of the assumption of taxonomic monism. That taxonomists choose to work on one map is a choice, not a necessity; most taxonomists believe it is a good choice, but it comes with necessary trade-offs, and these sometimes create valid, and unavoidable, disagreements.

\section{Valid disagreements over rank}

Valid disagreements over taxonomic rank occur when two taxonomists circumscribe the same taxa, but choose different ranks for the names. For example, one taxonomist may recognise a set of species as belonging to several subgenera within a single genus while another may recognise those subgenera as full genera, and allocate the species accordingly; or, one may circumscribe and name two distinct species while another may use the same circumscriptions but name them as two subspecies within a single species.

Disagreements over rank are a special class of disagreements over boundaries (circumscriptions). Thus, the taxonomist who circumscribes and names two species has employed narrower circumscriptions at species rank than the one who circumscribes a single species encompassing two recognised subspecies. And just as disagreements over circumscription usually reflect no more than different, and often perfectly valid, decisions taken by the taxonomists in abstracting the pattern of variation in nature, so too is choice of rank exactly that - a choice.

Disagreements over rank are particularly troubling at the taxonomic levels of genus and species (including subspecies, variety) and less so at higher ranks (families, orders etc.). Some argue that this is because the lower ranks are different in kind from the higher ranks (i.e., that species are 'special' taxa; see e.g. Ereshefsky, 1992). Practically, though, these disagreements are troublesome because they lead to alternate species names (because of the binomial naming system inherited from Linnaeus) whereas disagreements at high ranks do not.

\section{Valid disagreements between progress and stability}

In addition to the previously described disagreements rooted in taxonomic practice, another source of disagreement results from taxonomic tradition and taxonomic progress. Powerful new methods for capturing and analysing patterns of morphological, anatomical and genetic variation are being developed all the time, methods that of course were unavailable in the past. Taxonomy, like all scientific endeavours, is subject to revision in the face of new evidence and syntheses. At times, this leads to proposals to change existing, and sometimes well-established, taxonomic names and classifications based on the most recent analysis du jour. This is most striking in the context of the most important class of 'new' evidence - gene and genome sequences.

Opposing this tendency is a long-held tradition of stability in nomenclature and classification, resulting in a form of taxonomic and nomenclatural 'inertia'. While to some this may seem antithetical to scientific progress, there is important practical value behind this approach. A highly dynamic taxonomy not only leads to higher frequencies of the other kinds of disagreements discussed herein, but also threatens the informational bridges between and among biologically relevant information associated with taxa published over time. To the extent that taxonomy functions as a tool for practical communication, the efficacy of that communication becomes 
compromised if the labels and hierarchies used to organise and reference biodiversity are in constant flux. The value of stability in taxonomy is more or less based on the premise that important claims require robust evidence (i.e., that names and classifications should not be altered unless and until a compelling body of evidence sufficiently demonstrates that the benefits of the change outweigh the costs).

As with the other kinds of disagreements in taxonomy, there is no objectively 'right' or 'wrong' pathway for change and revision. The arguments that accepted taxonomy should follow the latest and most complete set of available evidence is every bit as legitimate as the arguments that taxonomy is devalued when it becomes excessively dynamic. Different practicing taxonomists find themselves at different points along the continuum between these two competing priorities, which leads to legitimate differences in taxonomic representations of the same sets of organisms.

Where disagreements do occur in taxonomy, they tend to follow a more or less predictable time-course. Alternate taxonomies are proposed, used and tested against criteria of utility and against new data. Taxonomic syntheses (revisions, field guides, floras etc.) are produced that weigh the competing views and gauge overall opinion. These syntheses tend to 'lock in' one or other of the alternatives for a time, until perhaps new data or new methods produce a new round of alternative taxonomies and the process repeats. This is analogous to the progressive testing and overall acceptance and rejection of hypotheses in other branches of science that deal with complex systems, or the growth in popularity of a useful map (one that 'makes sense' in the real world) over a less useful one.

As in other branches of science, a healthy balance is required between stability (or stasis) on one hand, and instability (or chaos) on the other. In the context of taxonomy and the management and curation of lists of accepted taxa, a healthy balance is needed between the desire for stability and the desire for accuracy in the face of growing knowledge.

\section{Why these disagreements won't go away}

Many disagreements between taxonomists - disagreements over species concepts, taxonomic circumscriptions and boundaries, taxonomic ranks and nomenclatural stabilityare often valid and do not point to an error or something being 'wrong'. Rather, taxonomic disagreements are a necessary consequence of the complexity of the domain and the nature of abstraction from the real world to the taxonomic 'map'. Like all models, maps are simplifications, and exactly how the simplifications are made will vary by context and author. Because of this, the disagreements cannot be easily and universally rectified by, for example, agreeing on a single species concept, using rule-based algorithms (such as a percentage of genetic difference) for delimiting taxa, or imposing strict rules rather than rules of thumb for choosing rank.

There are two main circumstances where the balance between disagreement and agreement tilts towards the former. One is the circumstance where many taxonomists work on one taxonomic group. The world's species can be usefully divided into those that are highly conspicuous to humans (usually because they have a body size and/or behaviours that bring them to attention) and those that are inconspicuous or cryptic. The former group includes most vertebrates (especially birds and mammals, and some reptile, amphibian and fish groups), vascular plants, a few insect groups such as butterflies and dragonflies, certain crustaceans, arthropods that serve as common disease vectors, a few charismatic groups of mushrooms and economically important fungi and a few of the more prominent marine phyla such as echinoderms and corals. Most other taxa fall into the 'rarely-noticed' group. Because the former group attracts more attention both from the public and from taxonomists, there is more opportunity for alternate views and disagreements. Simply put, if only one taxonomist works on a taxonomic group (and there are many taxonomic groups for which this is the case), then disagreement is unlikely.

The second circumstance where disagreements occur are cases where the patterns of variation are unusually complex. Evolution always produces complex patterns of variation, but some branches of the tree of life are stand-outs for complexity while others are more straightforward. Processes such as hybridisation, introgression, gene capture, rapid bursts of adaptive radiation and polyploidy all complicate the patterns of variation that taxonomists must deal with. Especially in cases when these two circumstances overlap, disagreement may, at times, be rife.

\section{Approaches to managing disagreements: agreed lists}

We show that taxonomic disagreements are a perpetual, inevitable and arguably healthy characteristic of biological taxonomy. However, an important tension exists between the need for a healthy and vibrant taxonomy, with its ensuing differences of opinion, alternative names, and ongoing name changes as taxonomic knowledge increases, and the need for an authoritative, relatively stable, scientifically robust taxonomic framework upon which others can build value in the biodiversity sciences. This is especially important because (through no fault of taxonomy itself) at times there are real-world consequences of taxonomic disagreements. For example, legislative conservation mechanisms in many countries use schedules of the names of taxa, and changing these schedules, when taxonomic disagreement results in name changes, is often cumbersome. Furthermore, some countries provide for the protection 
of species but not subspecies (meaning that disagreements over rank matters). These real-world consequences increase the need for agreement, but do not provide a clear way forward for resolving the disagreements.

One way of managing taxonomic disagreement is by creating and maintaining a global list of accepted taxa and their names, that is optimally fit-for-purpose for the very broad array of consumers outside the direct discipline of taxonomy, and that is managed and curated in the context of the real and unavoidable disagreements that characterise taxonomy.

A recent paper by Garnett et al. (2020) argued that the creation and curation of such a global list of the world's species and other taxa could provide substantial benefits for both taxonomy and its users, and would be an important way to operationalise the progressive acceptance of well-supported, useful, agreed taxonomic names, synonymies and classifications (and the occasional rejection of poorly supported ones). The paper recommended ten principles for the ongoing maintenance and governance of such a list. The first, and in some ways the most important, principle is that any species list must be based on science and be free from non-taxonomic considerations and interference. That is, taxonomy must continue largely as it currently is, unencumbered by formal regulation (see also Thomson et al., 2018), even if that leads to some degree of instability, disagreement and contention.

The papers in this series expand upon the themes and principles introduced in Garnett et al. (2020), and comprise attempts to explore and elucidate the necessary mechanisms, including checks and balance, needed to negotiate the inherent tension between the broad societal need for agreed lists of names and the needs of a relatively unencumbered science of taxonomy. Healthy disagreements and contention will continue as taxonomists continue to elucidate the rich and complex patterns of the diversity of life on Earth, and these disagreements should be seen as a strength rather than a weakness of the discipline; nevertheless, they need to be managed. The following papers explore aspects of effective management and governance for a system that yields a global list of accepted species and other taxa, for the benefit of users and practitioners of taxonomy.

Acknowledgements This paper has benefited from discussions with all members of the International Union of Biological Sciences (IUBS) Working Group on the Governance of Taxonomic Lists. We thank the IUBS for support to attend a meeting of the Working Group in Darwin, Australia, in 2020.

Data sharing Data sharing not applicable to this article as no datasets were generated or analysed during the current study.

Funding Discussions leading to this paper were part-supported through funding from the International Union of Biological Sciences (IUBS).

\section{Declarations}

Consent for publication All authors consent to this article being submitted for publication.

Conflict of interest The authors declare no competing interests.

\section{References}

Casadevall, A., \& Fang, F. C. (2008). Descriptive science. Infection and immunity, 76(9), 3835-3836.

Conix, S. (2018). Radical pluralism, classificatory norms and the legitimacy of species classifications. Studies in History and Philosophy of Science Part C: Studies in History and Philosophy of Biological and Biomedical Sciences, 73, 27-43. https://doi.org/10.1016/j.shpsc. 2018.11.002.

Dayrat, B. (2005). Towards integrative taxonomy. Biological journal of the Linnean society, 85(3), 407-417. https://doi.org/10.1111/j.10958312.2005.00503.x.

de Queiroz, K. (2007). Species concepts and species delimitation. Systematic Biology, 56(6), 879-886. https://doi.org/10.1080/ 10635150701701083.

Dupré, J. (1999). On the impossibility of a monistic account of species. In R. A. Wilson (Ed.), Species: New interdisciplinary essays (pp. $3-$ 22). Bradford Books.

Dupré, J. (2001). In defence of classification. Studies in History and Philosophy of Science Part C, 32(2), 203-219. https://doi.org/10. 1016/S1369-8486(01)s00003-6-.

Ereshefsky, M. (Ed.). (1992). The units of evolution: Essays on the nature of species. Journal of the History of Biology 25 (3):500-501.

Garnett, S. T., Christidis, L., Conix, S., Costello, M. J., Zachos, F. E., Buckeridge, J. S., Hobern, D., Thomson, S. A., van Dyck, P. P., Bánki, O. S., Bao, Y., Lien, A., Montgomery, N., Nikolaeva, S., Saroj, B., Whalen, A., Zhang, Z.-Q., \& Thiele, K. (2020). Principles for creating a single authoritative list of the world's taxa. PLOS One, 18(7), e3000736. https://doi.org/10.1371/journal.pbio.3000736.

Kendig, C., \& Witteveen, J. (2020). The history and philosophy of taxonomy as an information science. HPLS, 42, 40. https://doi.org/10. 1007/s40656-020-00337-8.

Kitcher, P. (1984). Species. Philosophy of science, 51(2), 308-333. https://doi.org/10.1086/289182.

Kitcher, P. (2001). Science, truth, and democracy. Oxford University Press.

Korzybski, A. (1933). Science and sanity. An introduction to nonAristotelian systems and general semantics, pp. 747-761. The International Non-Aristotelian Library Pub. Co..

McClure, C. J., Lepage, D., Dunn, L., Anderson, D. L., Schulwitz, S. E., Camacho, L., et al. (2020). Towards reconciliation of the four world bird lists: Hotspots of disagreement in taxonomy of raptors. Proceedings of the Royal Society B, 287(1929), 20200683. https:// doi.org/10.1098/rspb.2020.0683.

Mishler, B. D. (2009). Three centuries of paradigm changes in biological classification: Is the end in sight? Taxon, 58(1), 61-67 https://www. jstor.org/stable/27756824.

O'Hara, R. J. (1993). Systematic generalization, historical fate, and the species problem. Systematic Biology, 42, 231-246. https://doi.org/ $10.2307 / 2992462$.

Padial, J. M., Castroviejo-Fisher, S., Köhler, J., Vilà, C., Chaparro, J. C., \& De la Riva, I. (2009). Deciphering the products of evolution at the species level: The need for an integrative taxonomy. Zoologica Scripta, 38(4), 431-447. https://doi.org/10.1111/j.1463-6409.2008. 00381.x. 
Padial, J. M., Miralles, A., De la Riva, I., \& Vences, M. (2010). The integrative future of taxonomy. Frontiers in zoology, 7(1), 1-14. https://doi.org/10.1186/1742-9994-7-16.

Pante, E., Puillandre, N., Viricel, A., Arnaud-Haond, S., Aurelle, D., Castelin, M., Chenuil, A., Destombe, C., Forcioli, D., Valero, M., Viard, F., \& Samadi, S. (2015). Species are hypotheses: Avoid connectivity assessments based on pillars of sand. Molecular Ecology, 24(3), 525-544. https://doi.org/10.1111/mec.

Raposo, M. A., Stopiglia, R., Brito, G., Bockmann, F. A., Kirwan, G. M., Gayon, J., \& Dubois, A. (2017). What really hampers taxonomy and conservation? A riposte to Garnett and Christidis. Zootaxa, 4317(1), 179-184. https://doi.org/10.11646/zootaxa.4317.1.10.

Schlick-Steiner, B. C., Steiner, F. M., Seifert, B., Stauffer, C., Christian, E., \& Crozier, R. H. (2010). Integrative taxonomy: A multisource approach to exploring biodiversity. Annual review of entomology, 55, 421-438. https://doi.org/10.1146/annurev-ento-112408085432 .

Sluys, R. (2013). The unappreciated, fundamentally analytical nature of taxonomy and the implications for the inventory of biodiversity. Biodiversity Conservation, 22, 1095-1105. https://doi.org/10.1007/ s10531-013-0472-x.

Thomson, S. A., Pyle, R. L., Ahyong, S. T., Alonso-Zarazaga, M., Ammirati, J., Araya, J. F., Ascher, J. S., Audisio, T. L., AzevedoSantos, V. M., Bailly, N., Baker, W. J., Balke, M., Barclay, M. V. L., Barrett, R. L., Benine, R. C., Bickerstaff, J. R. M., Bouchard, P., Bour, R., Bourgoin, T., Boyko, C. B., Breure, A. S. H., Brothers, D. J., Byng, J. W., Campbell, D., Ceríaco, L. M. P., Cernák, I., Cerretti, P., Chang, C. H., Cho, S., Copus, J. M., Costello, M. J., Cseh, A., Csuzdi, C., Culham, A., D'Elía, G., d'Udekem d'Acoz, C., Daneliya, M. E., Dekker, R., Dickinson, E. C., Dickinson, T. A., van Dijk, P. P., Dijkstra, K. D. B., Dima, B., Dmitriev, D. A., Duistermaat, L., Dumbacher, J. P., Eiserhardt, W. L., Ekrem, T., Evenhuis, N. L., Faille, A., Fernández-Triana, J. L., Fiesler, E., Fishbein, M., Fordham, B. G., Freitas, A. V. L., Friol, N. R., Fritz, U., Frøslev, T., Funk, V. A., Gaimari, S. D., Garbino, G. S. T., Garraffoni, A. R. S., Geml, J., Gill, A. C., Gray, A., Grazziotin, F. G., Greenslade, P., Gutiérrez, E. E., Harvey, M. S., Hazevoet, C. J., He, K., He, X., Helfer, S., Helgen, K. M., van Heteren, A. H., Hita Garcia, F., Holstein, N., Horváth, M. K., Hovenkamp, P. H., Hwang, W. S., Hyvönen, J., Islam, M. B., Iverson, J. B., Ivie, M. A., Jaafar, Z., Jackson, M. D., Jayat, J. P., Johnson, N. F., Kaiser, H.,
Klitgård, B. B., Knapp, D. G., Kojima, J. I., Kõljalg, U., Kontschán, J., Krell, F. T., Krisai-Greilhuber, I., Kullander, S., Latella, L., Lattke, J. E., Lencioni, V., Lewis, G. P., Lhano, M. G., Lujan, N. K., Luksenburg, J. A., Mariaux, J., Marinho-Filho, J., Marshall, C. J., Mate, J. F., McDonough, M. M., Michel, E., Miranda, V. F. O., Mitroiu, M. D., Molinari, J., Monks, S., Moore, A. J., Moratelli, R., Murányi, D., Nakano, T., Nikolaeva, S., Noyes, J., Ohl, M., Oleas, N. H., Orrell, T., Páll-Gergely, B., Pape, T., Papp, V., Parenti, L. R., Patterson, D., Pavlinov, I. Y., Pine, R. H., Poczai, P., Prado, J., Prathapan, D., Rabeler, R. K., Randall, J. E., Rheindt, F. E., Rhodin, A. G. J., Rodríguez, S. M., Rogers, D. C., Roque, F. O., Rowe, K. C., Ruedas, L. A., Salazar-Bravo, J., Salvador, R. B., Sangster, G., Sarmiento, C. E., Schigel, D. S., Schmidt, S., Schueler, F. W., Segers, H., Snow, N., Souza-Dias, P. G. B., Stals, R., Stenroos, S., Stone, R. D., Sturm, C. F., Štys, P., Teta, P., Thomas, D. C., Timm, R. M., Tindall, B. J., Todd, J. A., Triebel, D., Valdecasas, A. G., Vizzini, A., Vorontsova, M. S., de Vos, J. M., Wagner, P., Watling, L., Weakley, A., Welter-Schultes, F., Whitmore, D., Wilding, N., Will, K., Williams, J., Wilson, K., Winston, J. E., Wüster, W., Yanega, D., Yeates, D. K., Zaher, H., Zhang, G., Zhang, Z. Q., \& Zhou, H. Z. (2018). Taxonomy based on science is necessary for global conservation. PLoS Biol., 16, e2005075. https://doi.org/10.1371/journal.pbio.2005075.

Wilkins, J. S. (2018). Species: The evolution of the idea, Second Edition. CRC Press.

Yeates, D. K., Seago, A., Nelson, L., Cameron, S. L., Joseph, L. E. O., \& Trueman, J. W. (2011). Integrative taxonomy, or iterative taxonomy? Systematic entomology, 36(2), 209-217. https://doi.org/10. 1111/j.1365-3113.2010.00558.x.

Zachos, F. E. (2016). Species concepts in biology: Historical development, theoretical foundations and practical relevance. Springer Nature.

Zachos, F. E., Christidis, L., \& Garnett, S. T. (2020). Mammalian species and the twofold nature of taxonomy: A comment on Taylor et al. 2019. Mammalia, 84(1), 1-5. https://doi.org/10.1515/mammalia2019-0009.

Publisher's note Springer Nature remains neutral with regard to jurisdictional claims in published maps and institutional affiliations. 